\title{
Investigations on Toxic Compound Traces in Powders and Sintered Ceramics
}

\author{
J.L. Longuet * \\ * CEA, DAM, LE RIPAULT, F-37260 Monts, France
}

The health protections needed for the personals who use some chemical products, form manufacturing to recycling, are set by the risks these products bring. Annex I of Directive 67/548/EEC contains a list of harmonized classification and labeling for substances or groups of substances, which are legally binding within the EU. For example nickel oxide (NiO) is classified as toxic and carcinogenic-mutant-damaging for reproduction (CMR). To classify CMR a preparation, EU directive 04-11-09 set a threshold concentration of 0.1\%wt. of free $\mathrm{NiO}$ in the material.

The aim of this talk is to present an original method developed in our laboratory to detect free $\mathrm{NiO}$ traces in ferrite-like ceramics. Characterizations have been carried out at different stages along the elaboration process (figure 1) and in the environment area of work.

Firstly, X-ray diffraction (XRD) and atomic emission spectroscopy ion coupled plasma (AESICP) have been carried out. A structural interference between two components restrain XRD from concluding, even in presence of a high amount of free $\mathrm{NiO}$ in the sample. No selective way of dissolution of nickel was found in the $\mathrm{NiO}$ and in the final material to permit to solve the problem.

Finally, a specific methodology was developed from local analysis by electron probe microanalysis (EPMA, CAMECA SX50 equipped with four wavelength dispersive spectrometers (WDS)). The basis of the method is to take advantage about the large difference in nickel content between the two components (figure 2). With EPMA, it is possible to detect local variation of compositions within a few microns of resolution. X-ray maps are recorded on polished surface of resin embedded material (figures 3 and 4). With 40 analysis zones, 80\% of the whole surface (300 $\mathrm{mm}^{2}$ ) can be investigated in a reasonable time with a spatial resolution adapted to the microstructure (figure 5). Then, post treatment of the X-ray maps is made (figure 6) to detect free $\mathrm{NiO}$ and quantify a $\mathrm{NiO}$ surface rate. Detection limit of the method can be determined as the detection of a single pixel above the threshold set to separate $\mathrm{NiO}$ and ferrite. Regarding the area scanned, this level is suitable with the detection threshold needed by the EU directives.

After testing the method on $0.1 \%$ wt $\mathrm{NiO}$ standard, samples from different steps of the elaboration process and from atmospheric environment were characterized. In some samples, $\mathrm{NiO}$ traces were detected but never above the threshold limit imposed by the EU directives. 


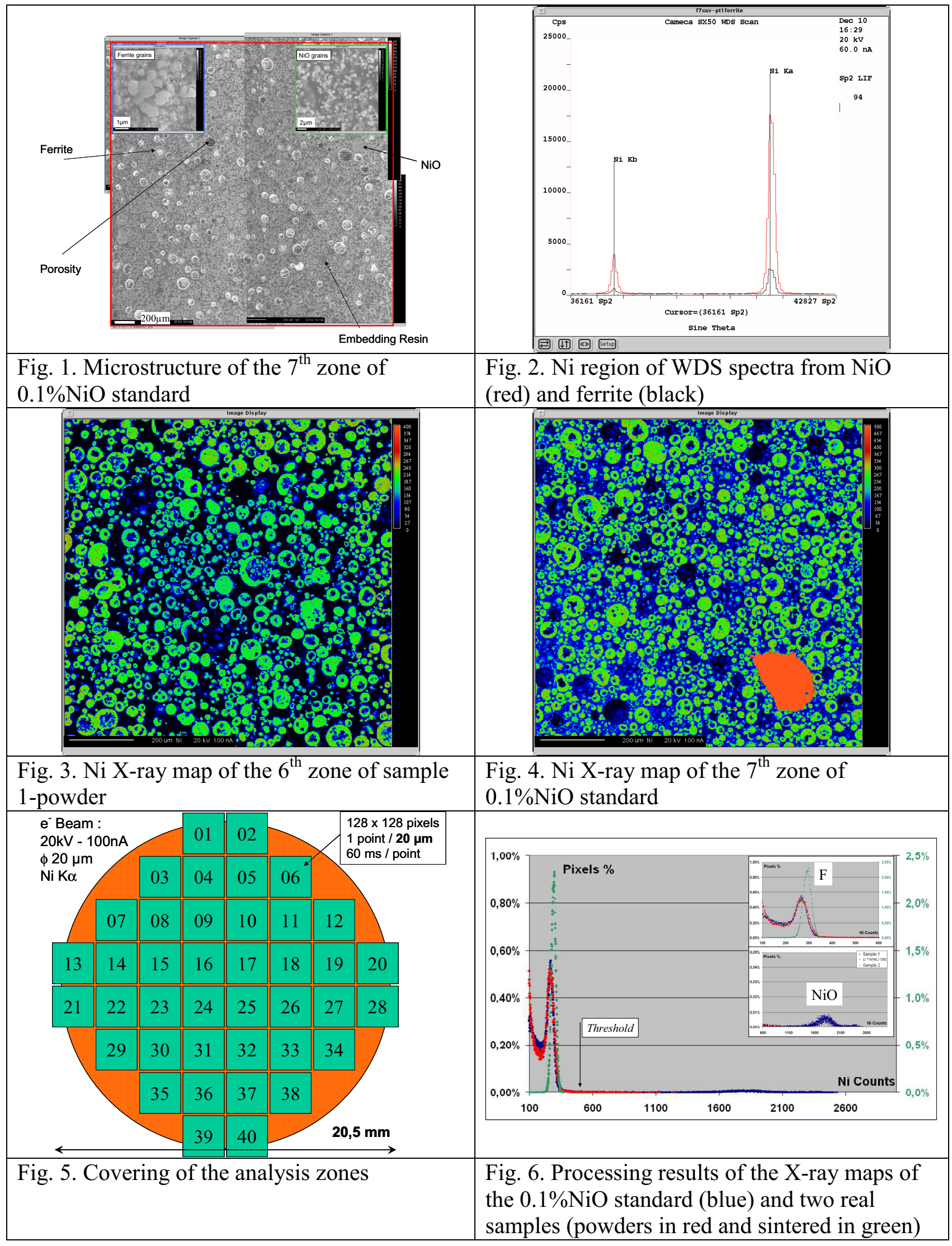

